\title{
Zuska's breast disease: Breast imaging findings and histopathologic overview
}

\author{
Luis F Serrano, María M Rojas-Rojas, Fedel A Machado \\ Department of Radiology, Hospital Universitario Fundación Santa Fe de Bogotá, 'Department of Medicine, School of Medicine, \\ Universidad de los Andes, Bogotá, Colombia
}

Correspondence: Dr. Luis F Serrano, Department of Radiology, Hospital Universitario Fundación Santa Fe de Bogotá, Bogotá, Carrera 7 No.117-15. Colombia. E-mail: monoserrano@gmail.com

\begin{abstract}
Zuska's disease describes the clinical condition of recurrent central or periareolar nonpuerperal abscesses associated with lactiferous fistulas. Pathogenesis involves the occlusion of an abnormal duct through an epithelial desquamation process that causes ductal dilatation, stasis of secretions, and periductal inflammation. Patients with Zuska's disease may develop chronic draining sinuses near the areola from lactiferous ducts fistula; therefore, the underlying abnormal duct system must be located and excised for proper treatment. Zuska's disease is often misdiagnosed and mistreated and is associated with significant morbidity, including the recurrence of abscess and cutaneous fistula formation. This case series aimed to help clinicians investigate and manage this disorder. The clinical and imaging findings, histopathologic correlation, and treatment of Zuska's disease are discussed.
\end{abstract}

Key words: Abscess; breast diseases; lactiferous fistula; Zuska's disease

\section{Introduction}

Zuska's disease describes the clinical condition of recurrent central or periareolar nonpuerperal abscesses associated with lactiferous fistulas, representing $1-2 \%$ of all symptomatic breast processes. ${ }^{[1,2]}$ This disease typically occurs in smokers with a mean age of presentation of 47 years old. ${ }^{[3,4]}$ This disease can present with a wide variety of symptoms and unfamiliar findings that can delay diagnosis and treatment [Table 1]. ${ }^{[1,5,6]}$ Complete excision of the fistula and the abnormal duct is recommended to prevent recurrent episodes. ${ }^{[7]}$ Our case series of four Zuska's disease cases exemplify the diagnostic and therapeutic challenges of this condition, including breast imaging findings and histopathologic correlation.

\begin{tabular}{|l|l|}
\hline \multicolumn{2}{|c|}{ Access this article online } \\
\hline Quick Response Code: & \\
\cline { 1 - 2 } & Website: \\
& www.ijri.org \\
\cline { 2 - 3 } & DOI: \\
\hline
\end{tabular}

\section{Materials and Methods}

Clinical imaging of the breast was performed in all patients by mammogram and ultrasound evaluation. In selected cases, a computed tomography (CT) and positron emission tomography (PET) scan, performed for other reasons, aided the diagnosis. Biopsies were taken by ultrasound-guided core needle biopsy, fine needle aspiration (FNA), or both. Tissue analysis was done with hematoxylin and eosin (H\&E) stain, while cytologic analysis was done with Papanicolaou stain.

This is an open access journal, and articles are distributed under the terms of the Creative Commons Attribution-NonCommercial-ShareAlike 4.0 License, which allows others to remix, tweak, and build upon the work non-commercially, as long as appropriate credit is given and the new creations are licensed under the identical terms.

For reprints contact: WKHLRPMedknow_reprints@wolterskluwer.com

Cite this article as: Serrano LF, Rojas-Rojas MM, Machado FA. Zuska's breast disease: Breast imaging findings and histopathologic overview. Indian J Radiol Imaging 2020;30:327-33.

Received: 02-Apr-2020 Accepted: 27-May-2020

Revised: 03-May-2020 Published: 15-Oct-2020 
Table 1: Key features of Zuska's disease

\begin{tabular}{ll}
\hline Disease & Features \\
\hline Symptoms & Breast pain/lump, nipple discharge, and areolar pain/lump. \\
Clinical & $\begin{array}{l}\text { Palpable breast mass, tenderness, erythema, warmth, breast } \\
\text { findings }\end{array}$ \\
swelling, skin thickening, and/or axillary lymphadenopathy. \\
Pathogens & $\begin{array}{l}\text { Staphylococcus aureus, Pseudomonas aeruginosa, Proteus } \\
\text { mirabilis. }\end{array}$ \\
Differential & Inflammatory carcinoma, atypical infection, fibroadenoma. \\
Diagnosis &
\end{tabular}

Based on references ${ }^{[1,5,6]}$

\section{Results}

The study population consisted of four patients with Zuska's disease diagnosis. Age at diagnosis ranged from 36 to 55 years. Two patients had a smoking history and three out of four patients had a family history of breast cancer. Three out of four patients had multiple episodes before diagnosis and the presented symptoms included palpable mass (two cases), draining abscess (one case,) and increased nipple sensitivity (one case).

Imaging evaluation included mammogram studies in all patients and three out of four patients had ultrasound studies before biopsy. In two cases, additional studies, performed for other reasons (liver mass study and laryngeal cancer follow-up), were included, including CT and PET.

Combined FNA and core needle biopsy were performed in two cases. Among the remaining two cases, 1 had FNA and 1 case had a core needle biopsy. Two of the patients were treated with drainage and antibiotic treatment presenting with recurrence and two of the patients were treated with surgery with no recurrence on follow up.

\section{Case 1}

A 55-year-old African-American single female, with high school equivalent education and a 30 pack-year smoking history, presented with a one-week history of a right breast lump and green nipple discharge. On physical examination, a large palpable non-fixed mass near the right areola was present. The mass was tender to palpation and there were no palpable axillary lymph nodes. The patient was being treated for laryngeal cancer at the time of presentation and had a family history of breast cancer with her mother dying at age 62 .

A recent $\mathrm{CT}$ of the chest incidentally showed a $3.2 \mathrm{~cm} \times 3.2 \mathrm{~cm}$ right retroareolar, lobulated breast mass with mixed attenuation coefficients [Figure 1]. Her most recent mammogram performed one month prior to her presentation showed a subareolar breast mass, with skin thickening and subtle retraction of the nipple/ areolar complex [Figure 2]. This study was classified as BI-RADS 0 with a recommendation for ultrasound, which revealed a well-circumscribed, oval subareolar mass with

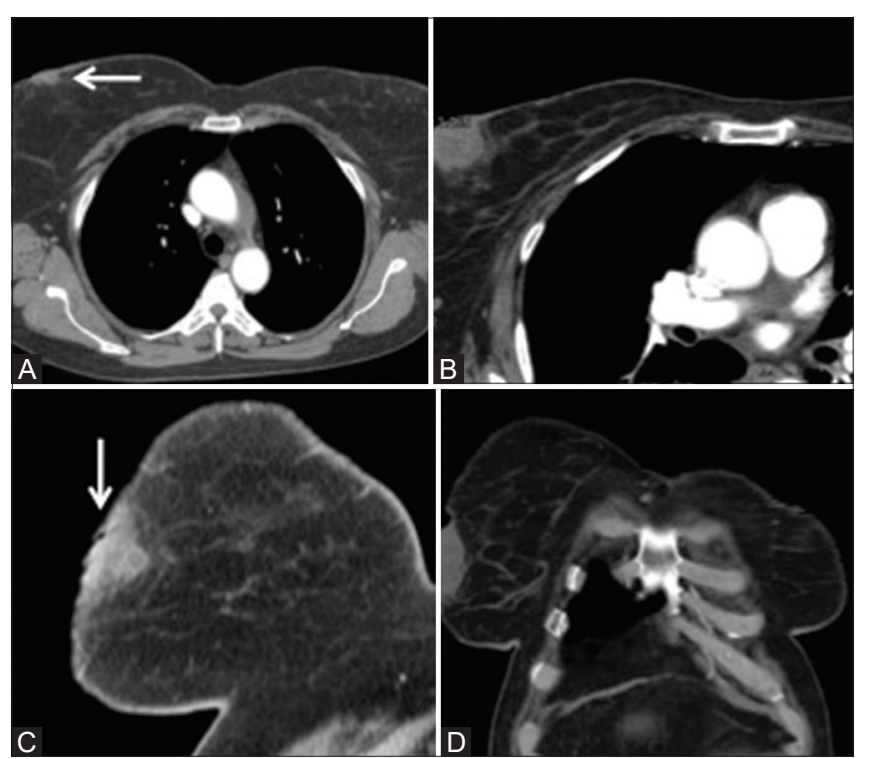

Figure 1 (A-D): Case 1. Contrast-enhanced CT images. (A and B) Axial plane. (C and $D)$ Coronal plane. Arrows show a right retroareolar lobulated hyperdense mass measuring $3.2 \mathrm{~cm} \times 3.2 \mathrm{~cm}$

heterogeneous echotexture, with both cystic and solid components, abutting the skin. No increased vascularity was found on power Doppler mode. Along with this mass, some dilated ducts were found with no cleavage planes [Figure 3]. In addition, a PET scan was performed for laryngeal cancer follow-up, showing a right breast mass with increased uptake with a maximum standardized uptake value (SUV) of 4.3 [Figure 4 ].

To rule out malignancy, FNA and ultrasound-guided core biopsy with a vacuum-assisted device using a 10-gauge needle were performed [Figure 5]. The H and E stain showed lamellated keratinous debris and acute inflammatory infiltrate, consistent with Zuska's breast disease. The drainage of the areolar area was performed and the abscess was packed. The patient was sent home with trimethoprim/ sulfamethoxazole treatment and a one-week follow-up appointment was scheduled. She returned eight months later with persistence of the abscess and was treated once again with incision and drainage. She was sent home on cephalexin and given a follow-up appointment, at which point she was lost to follow up.

\section{Case 2}

A 51-year-old African-American married woman, with secondary school education, and a smoking history of 0.5 packs of cigarettes daily, had a one-week history of draining right breast abscess. This patient had a similar episode 1.5 years before and was treated at another facility. Physical exam showed an open draining wound on the areola with a firm surrounding area of induration measuring $4 \times 2 \mathrm{~cm}$. A mammogram was recommended. In addition, the patient was scheduled for an outpatient abdomen CT for palpable liver mass, showing a 

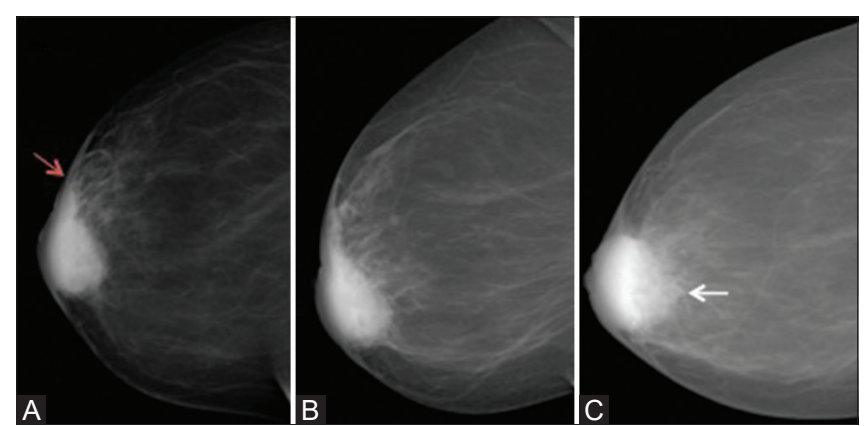

Figure 2 (A-C): Case 1. Right breast mammogram. (A) Craniocaudal view (CC). (B) Mediolateral oblique view (MLO). (C) Lateral view. Retroareolar lobulated dense mass (White arrow) with skin thickening (Red arrow)
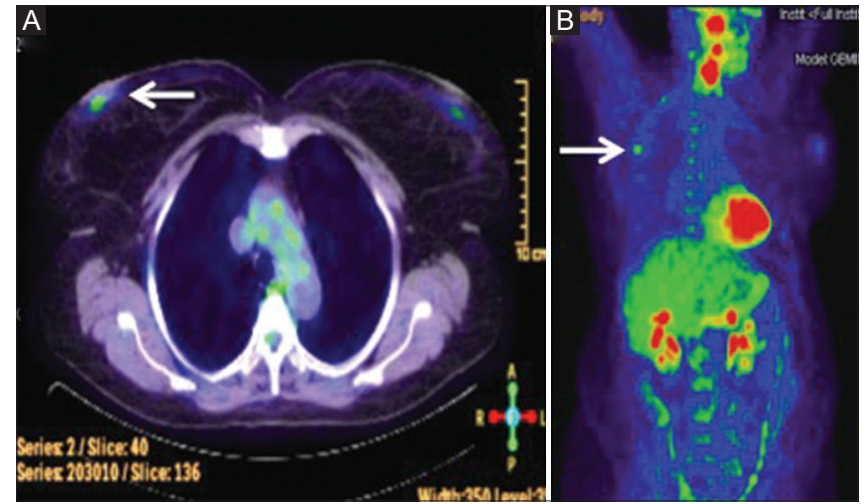

Figure 4 (A and B): Case 1. PET scan. (A) Axial (B) Coronal. Right breast mass with increase uptake

retroareolar right breast mass [Figure 6]. Moreover, no liver masses were identified.

The mammogram showed slight asymmetry in the central anterior third of the right breast with no other associated mammographic findings, classified as BIRADS-0 [Figure 7]. The next exam performed was an ultrasound that revealed a retroareolar, hypoechoic, irregular, $3 \times 1 \mathrm{~cm}$ sonographic abnormal area, overlying mildly dilated ducts, with no cleavage planes, concordant with mammographic findings, [Figure 8]. An ultrasound-guided core biopsy evaluation requested by her clinician was performed and the pathology results were consistent with Zuska's breast disease [Figure 9].

On follow-up, this patient has continued to struggle with chronic breast abscesses, presenting to the ER and breast clinic repeatedly with only temporary remission achieved by drainage and antibiotic treatment. Over the past 30 months, she had received five mammograms and two ultrasound-guided biopsies.

\section{Case 3}

A 36-year-old female patient presented with left breast pain, a palpable lump, and skin thickening in the areolar region. The changes have been present for the past four

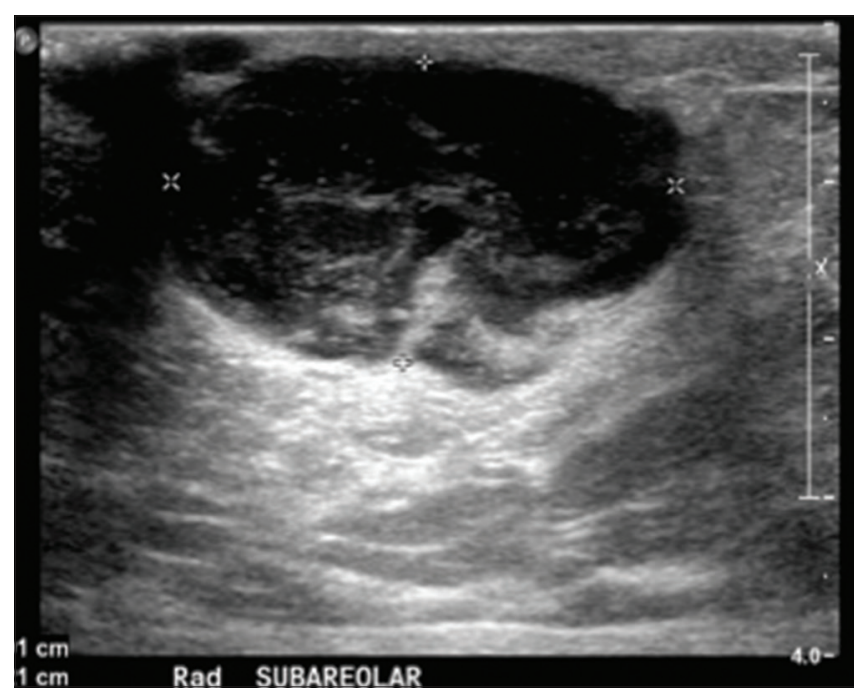

Figure 3: Case 1. Right breast ultrasound. Subareolar lobulated well-circumscribed mass with internal echoes

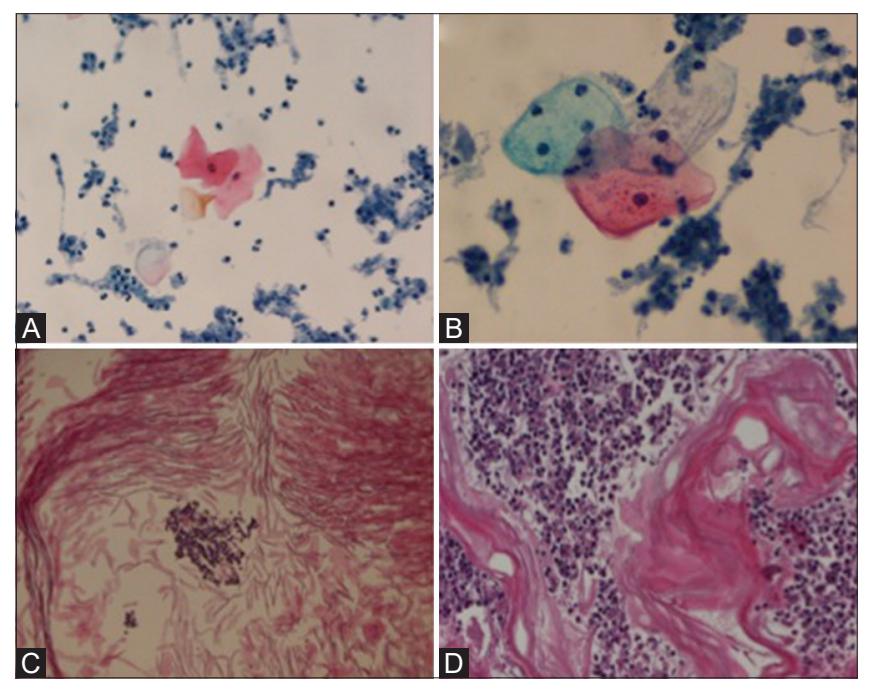

Figure 5 (A-D): Case 1. Histopathological examination. (A) FNA, filter preparation, Papanicolaou stain $200 \times$ magnification, mature squamous cells, and acute inflammatory exudate. (B) FNA, filter preparation, Papanicolaou stain, 400x magnification, mature squamous cells, and acute inflammatory exudate. (C) FNA, cell block preparation, $\mathrm{H}$ and $\mathrm{E}$ stain, 200x magnification, lamellated keratinous debris, and acute inflammatory exudate. (D) Core needle biopsy, $\mathrm{H}$ and $\mathrm{E}$ stain, 100x magnification, lamellated keratinous debris, and acute inflammatory exudate

years, but have worsened in the past two weeks. The mass shrinks and swells with no relation to her menstrual cycle. The patient denies trauma to her breasts and has a family history of a maternal aunt diagnosed with breast cancer at age 60 . On physical exam, there is a large superficial mobile mass in the left areola area, which is tender, red, and warm [Figure 10].

A mammogram was performed showing retroareolar asymmetry with areolar and skin thickening [Figure 11]. Left breast ultrasound revealed a large hypoechoic irregular mass beneath the skin with marked skin thickening and a 


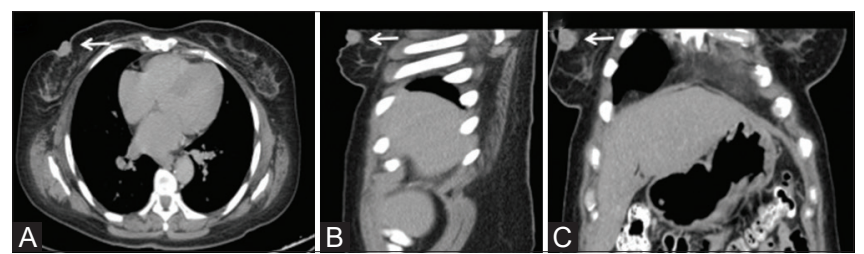

Figure 6 (A-C): Case 2. Abdomen CT performed for liver mass evaluation showing a right retroareolar breast mass. (A) Axial (B) Sagittal (C) Coronal
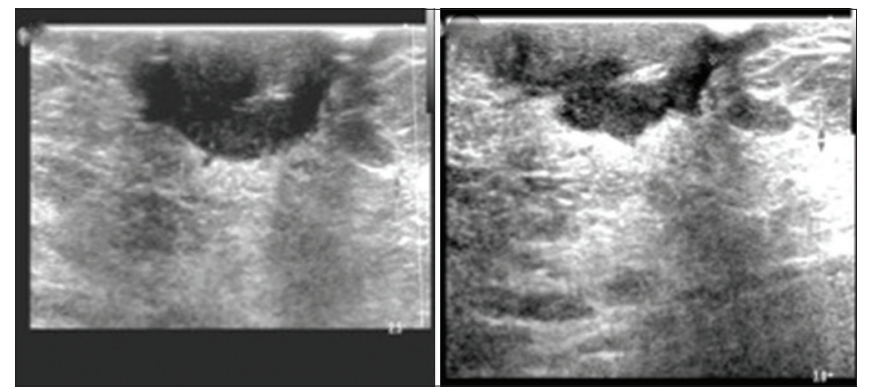

Figure 8: Case 2. Right breast ultrasound. Multilobulated heterogeneous retroareolar mass with internal echoes

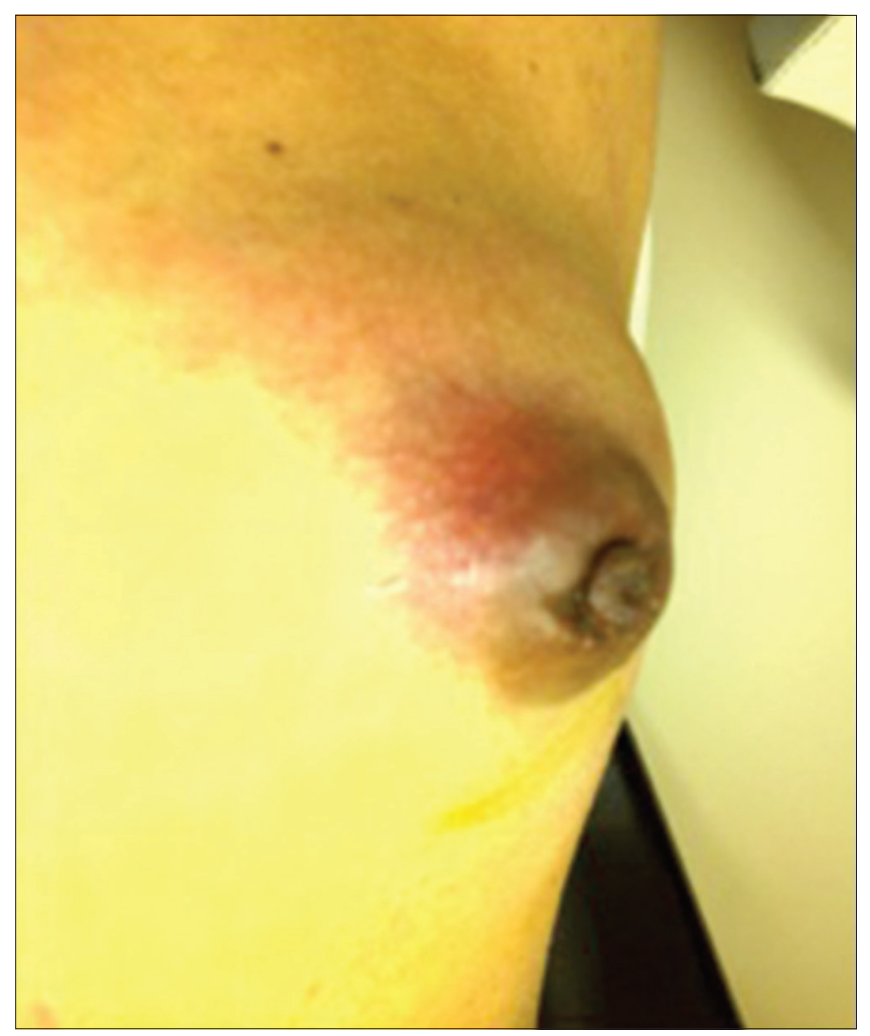

Figure 10: Case 3. Large superficial mobile mass in the left areola

mild increase in peripheral vascularity on color Doppler images [Figure 12]. A needle aspiration biopsy and a core needle biopsy were performed. Histopathological analysis revealed mature squamous cells, keratinous material with mixed inflammatory infiltrate and abundant foamy macrophages [Figure 13]. A diagnosis of Zuska's disease

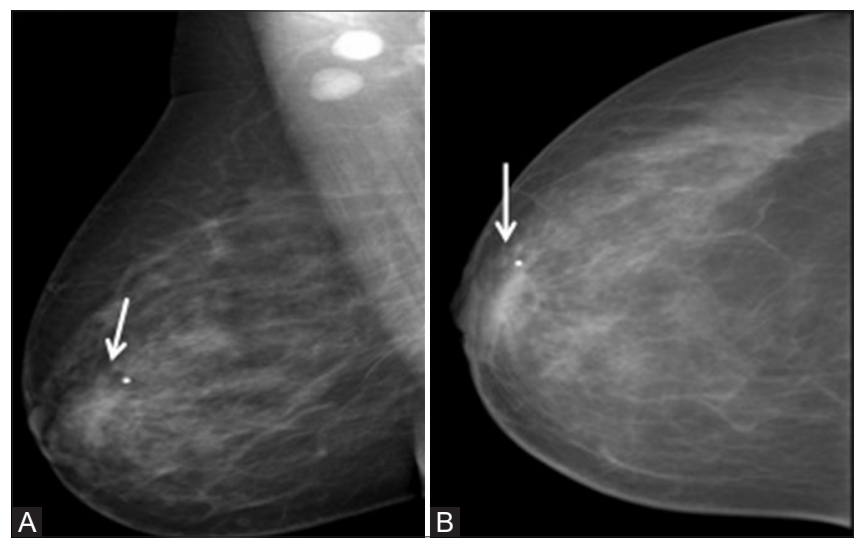

Figure 7 (A and B): Case 2. Right breast mammogram. (A) MLO and (B) CC views, retroareolar asymmetric density and abnormal rounded dense lymph nodes in the ipsilateral axilla

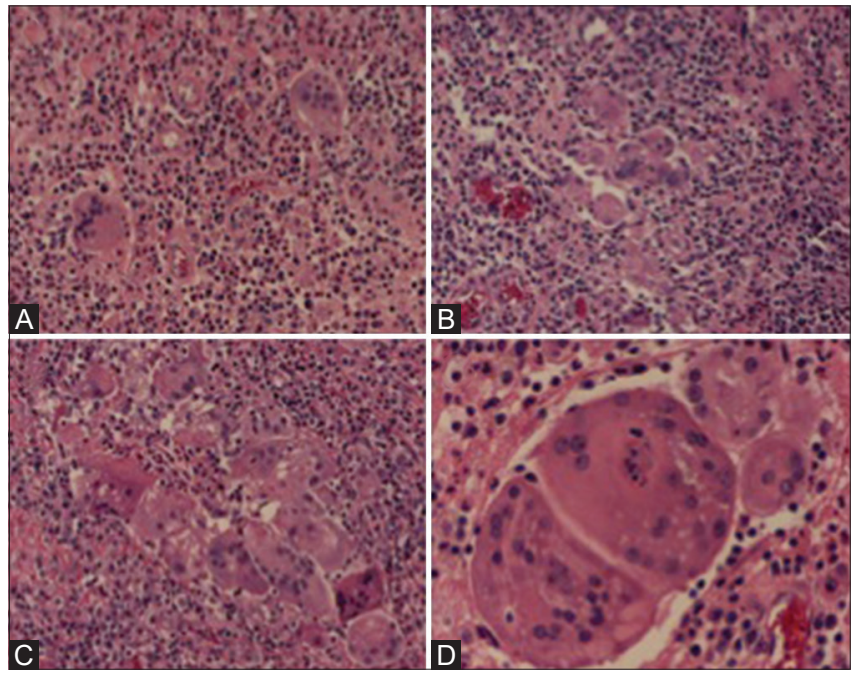

Figure 9 (A-D): Case 2. Biopsy $\mathrm{H}$ and $\mathrm{E}$ stain. (A) 100X magnification. (B-C) $200 \mathrm{X}$ magnification. (D) 400X magnification. Mixed inflammatory infiltrates and foreing-body-type multinucleated giant cells
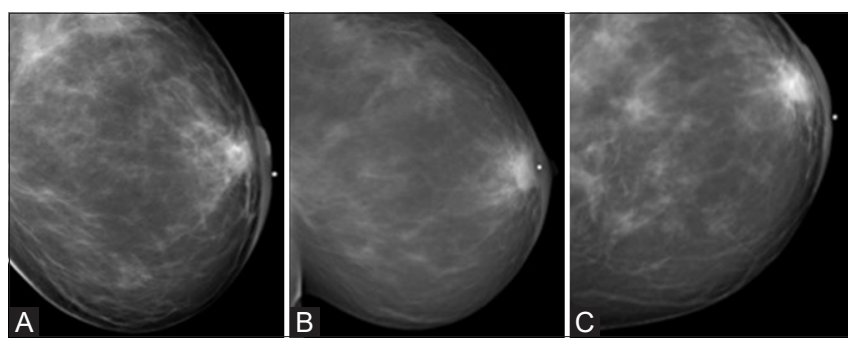

Figure 11 (A-C): Case 3. Left breast mammogram. (A) CC, (B) MLO, and $(C)$ Lateral views. Retroareolar asymmetry with areolar and skin thickening

was made and the patient was referred to plastic surgery for excisional treatment. There was no recurrence on follow-up.

\section{Case 4}

A 46-year-old female patient presented with increased nipple sensitivity in her right breast with no other associated 
symptoms. The patient had a history of mastitis after the birth of her first child and her mother was treated for breast cancer at age 70. Physical exam showed symmetrical breasts with slightly inverted nipples and there were no skin changes or dominant masses, but fibrocystic changes were present bilaterally.

A mammogram performed at an outside clinic showed skin thickening in the right breast and ultrasound demonstrated a $2 \times 1 \mathrm{~cm}$ oval mass with circumscribed
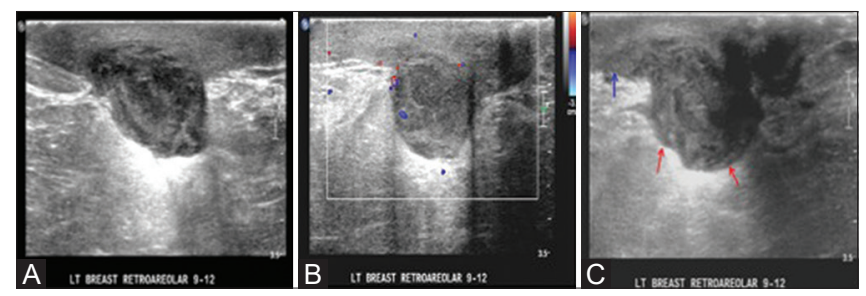

Figure 12 (A-C): Case 3. (A) Left breast ultrasound reveals a large hypoechoic irregular mass. (B) There is a mild increase in peripheral vascularity on color Doppler images. (C) Hypoechoic irregular mass (red arrows) beneath the skin with marked skin thickening (blue arrows)
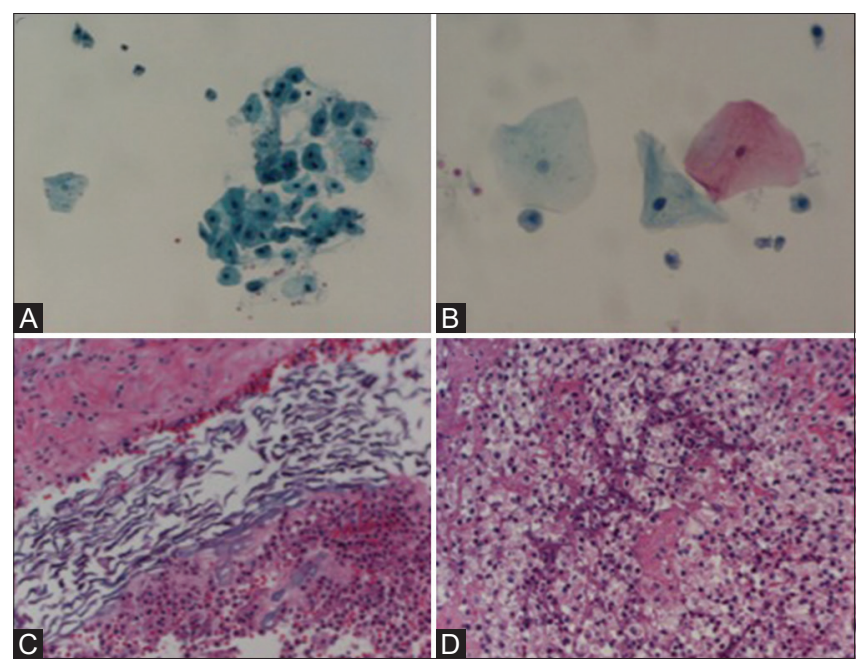

Figure 13 (A-D): Case 3. (A) FNA, filter preparation, Papanicolaou stain, $200 \times$ magnification, mature squamous cells, and scant neutrophils. (B) FNA, filter preparation, Papanicolaou stain, 400× magnification, mature squamous cells, and scant neutrophils. (C) Biopsy, $\mathrm{H}$ and $\mathrm{E}$ stain, $200 \times$ magnification, keratinous material and acute inflammatory infiltrate. (D) Biopsy, $\mathrm{H}$ and $\mathrm{E}$ stain, 200x magnification, and mixed inflammatory infiltrate with abundant foamy macrophages
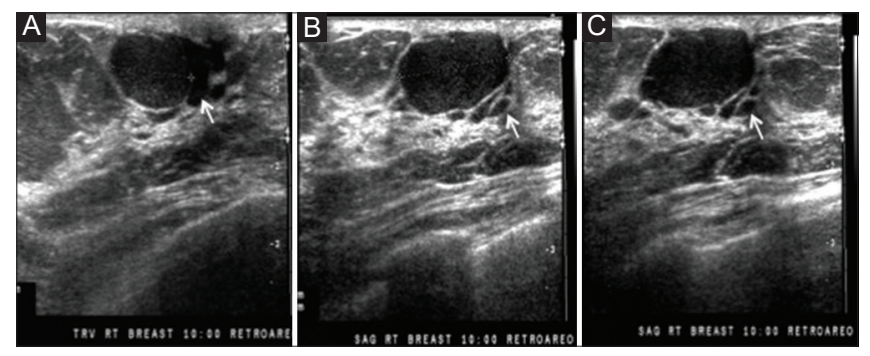

Figure 14: Case 4. (A-C) Right breast ultrasound shows a wellcircumscribed oval mass with dilated ducts (white arrows on A-C) margins and ductal dilatation [Figure 14]. Accordingly, an ultrasound-guided fine-needle aspiration using an $18 \mathrm{G}$ needle was performed and 2 cc of viscous yellow fluid was sent for cytological examination. Histological evaluation demonstrated abundant amorphous debris, cholesterol crystals, polymorphonuclear leukocytes, and atypical cells, consistent with Zuska's disease. The patient underwent a needle-localized excisional biopsy of the right breast [Figure 15]. Histopathological analysis showed mammary duct ectasia with mild acute and chronic inflammation. There was no evidence of atypia or malignancy. The patient was discharged home and remains asymptomatic on follow-up.

\section{Discussion}

The pathogenesis of Zuska's disease involves squamous metaplasia of the cuboidal epithelium lining the lactiferous ducts. The squamous lining produces large amounts of keratin that obstructs and dilates the ducts, leading to acute inflammatory infiltrates and cellular debris. These ducts become secondarily infected as a result of stasis and bacterial invasion, which leads to abscess formation. The abscess may drain spontaneously and can develop into a periareolar cutaneous fistula. ${ }^{[8]}$ According to one retrospective study conducted by $\mathrm{Fu}$ et al., 25-40\% of patients with Zuska's disease develop recurrent abscesses and $33 \%$ demonstrate cutaneous fistula formation. ${ }^{[9]}$ In our cases, two-fourth of the patients presented with recurrent abscesses following drainage treatment.

Several hypotheses have been suggested to explain the cause of squamous metaplasia, including a congenital anomaly of the lactiferous ducts, a complication of comedomastitis, nipple retraction, and a relative state of vitamin A deficiency. ${ }^{[5]}$

Patients with diabetes mellitus, HIV infection, or those on immunosuppression therapy are at increased risk for developing breast infections. Diabetes mellitus is strongly associated with breast abscesses in nonlactating women. Tobacco smoking is associated with the development of primary abscess including subareolar abscess, and smokers are more likely to develop recurrent episodes. Smoking is thought to have a direct toxic effect on the retroareolar ductal epithelium or an indirect effect via the hormonal stimulation of breast secretion, thus predisposing to Zuska's disease. Also, the plasma levels of $\beta$-carotene (Vitamin A precursor) are lower among smokers explaining why squamous metaplasia occurs more frequently. ${ }^{[5]}$ Among our cases, two patients reported tobacco use.

Other risk factors for abscess development include nipple piercing, hair removal from the areola, and nipple cracks or fissures. ${ }^{[5]}$ 


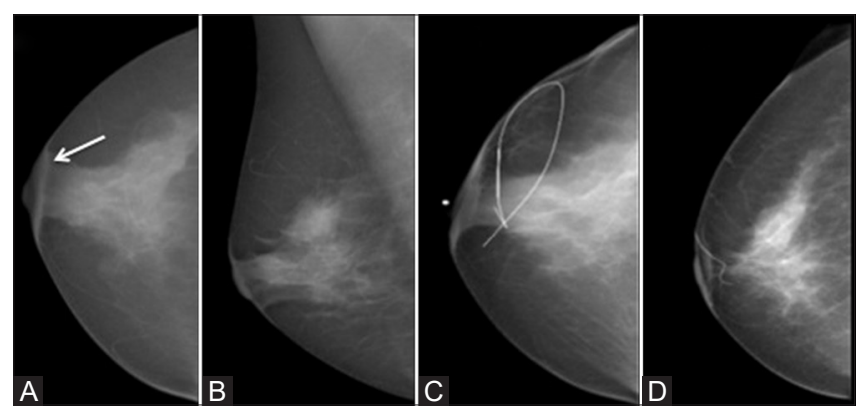

Figure 15 (A-D): Case 4. Right breast mammogram. (A) CC and (B) MLO views. Subareolar skin thickening (arrow). The mass is obscured by dense glandular tissue. (C) Needle wire localization (D) Postoperative (mass removed, scar marker)

It may be difficult to distinguish between mastitis and a breast abscess clinically. Once a breast mass or area of tenderness is noted it is recommended to perform an ultrasound. An abscess appears as a hypoechoic collection of varying sizes and shapes, usually multiloculated, with a thick peripheral echogenic area of increased vascularity. In contrast, mastitis presents with an ill-defined area of altered echotexture, increased echogenicity in the fat lobules, hypoechoic areas in the glands, and skin thickening [Table 2] ${ }^{[2,6,6,10]}$ In our cases, ultrasound findings were indicative of breast abscess.

It may also be difficult to determine if a breast mass in a nonlactating woman is an abscess when signs of inflammation are absent. In this case, a combination of mammographic and sonographic features can help guide appropriate management. A mammogram is recommended to help exclude a malignant process, especially in women over 30 years old. In the case of Zuska's disease, mammograms may reveal skin thickening, asymmetric density, ill-defined mass, and/or architectural distortion. Unfortunately, these findings can resemble malignancy. Therefore, any inflammatory mass that does not respond to treatment should be biopsied to exclude malignancy. ${ }^{[10]}$ In our cases, breast-imaging findings, including mammography and ultrasound, along with histopathology results confirmed Zuska's disease diagnosis.

Aspiration and drainage has been advocated for breast abscesses. ${ }^{[1-13]}$ Hook et al. assessed ultrasound-guided percutaneous drainage of breast abscesses in 13 patients and found seven cases with abscesses $2.4 \mathrm{~cm}$ or smaller, that were completely drained or aspirated and resolved without surgery. However incision and drainage is necessary for definitive treatment in partially drained abscesses larger than $3 \mathrm{~cm}^{[12]}$

Giess et al. Also evaluated aspiration of breast abscesses and found that abscesses associated with lactation or breast biopsy could be managed with aspiration, even
Table 2: Imaging features of Zuska's disease

Imaging Modality Imaging Findings

Mammogram Irregular mass, focal asymmetric density, diffuse asymmetric density, circumscribed mass, and architectural distortion, skin thickening (diffuse or focal)

Ultrasound

CT

Well-circumscribed masses or irregular lesions, with poor heterogeneous internal echoes; in some cases, fine fistulous tracts can be identified; skin thickening is a very common associated finding.

Most of the breast lesions detected under this modality are incidental, since CT is not commonly used as a breast imaging modality. Imaging can show abnormal retroareolar density, irregular mass with architectural distortion, nipple inversion and skin thickening.

MRI

Irregular mass, inverted nipples, abscess cavities and fistulas. Findings on unenhanced T1-weighted images are nonspecific, while postcontrast $\mathrm{T} 1$ images showed irregular heterogeneous enhancing areas consistent with a subareolar collection, with a benign type curve of progressive enhancement pattern. The role of kinetics on MRI is an important tool in the differential diagnosis of benign and suspicious lesions in patients with Zuska's.

Based on references ${ }^{[2,6,9,10]}$

when large. This procedure allows the resolution of acute inflammation and helps tailor antibiotic therapy. However, surgical intervention is recommended in chronic cases with recurrence of fistula ${ }^{[14]}$ Among our four cases, two patients were treated with drainage and antibiotic treatment presenting multiple recurrent episodes on follow-up. These patients should be recommended for surgical excision of the abnormal lactiferous duct and abscess cavity.

The definitive treatment for Zuska's breast disease should include broad-spectrum antibiotic therapy and the removal of the infected obstructed lactiferous ducts, the affected ampulla, and the fistula if present. ${ }^{[15]}$ Atkins first recommended abnormal ductal system excision in 1955, followed by Patey in 1958 and Livingston in 1962 who reported ten cases of breast ductal fistula treated with excision of the entire abnormal duct and its associated fistula, presenting with no recurrences on follow-up. ${ }^{[3]}$ Among our cases, two patients were treated with excisional treatment without evidence of recurrence on follow-up.

Differential diagnoses should be considered in refractory cases, including deep or multiple abscesses, mastitis, fistula formation, atypical infection, or malignancy. ${ }^{[6]}$ Peripheral non-puerperal abscesses are less common than central abscesses and usually occur in older women with chronic underlying medical conditions. This type of abscess responds well to drainage and antibiotics, and recurrence is rare. Granulomatous mastitis is a rare benign inflammatory breast disease of unknown etiology that develops within 5 years of lactation. At mammography, common imaging findings include asymmetry, masses, or increased parenchymal density. Ultrasound shows parenchymal distortion or multiple heterogeneous ill-defined hypoechoic 
masses with tubular hypoechoic extensions across the breast lobules. In these cases, tissue sampling is needed to exclude malignancy and confirm the diagnosis. Treatment involves antibiotics and steroid therapy and recurrence is common. Inflammatory breast carcinoma accounts for $2-5 \%$ of all cancers and is a highly aggressive form. Mammography and biopsy should be considered if there is no response within 1-2 weeks after an initial trial of antibiotics. Mammographic features include diffusely increased breast density and skin and trabecular thickening. Ultrasound usually shows skin thickening, loss of the plane between the dermis and the subcutaneous fat, hypoechoic appearance of the subcutaneous fat and increased echogenicity of breast tissue due to edema. Also a focal solid mass may be identified. MRI may help differentiate inflammatory carcinoma form mastitis and determine the extent of the disease. Treatment includes chemotherapy, modified radical mastectomy and radiotherapy. ${ }^{[6]}$

Finally, Zuska's disease is an uncommon benign entity that is often misdiagnosed and mistreated and this study confirms that surgical treatment is indicated in order to avoid recurrence.

\section{Declaration of patient consent}

The authors certify that they have obtained all appropriate patient consent forms. In the form the patient(s) has/have given his/her/their consent for his/her/their images and other clinical information to be reported in the journal. The patients understand that their names and initials will not be published and due efforts will be made to conceal their identity, but anonymity cannot be guaranteed.

\section{Financial support and sponsorship}

Nil.

\section{Conflicts of interest}

There are no conflicts of interest.

\section{References}

1. Kasales, CJ, Han B, Smith JS, Chetlen AL, Kaneda HJ, Shereef S. Nonpuerperal mastitis and subareolar abscess of the breast. Am J Roentgenol 2014;202:W133-9.

2. Trop I, Dugas A, Davis J, El Khoury M, Boileau JF, Larouche N, et al. Breast abscesses: Evidence-based algorithms for diagnosis, management, and follow-up. Radiographics 2011;31:1683-99.

3. Zuska JJ, Crile G, Ayres WW. Fistulas of lactifierous ducts. Am J Surg 1951;81:312-7.

4. Livingston SF, Arlen M. Ductal fistula of the breast. Ann Surg 1962;155:316-9.

5. Gollapalli V, Liao J, Dudakovic A, Sugg SL, Scott-Conner CE, Weigel RJ. Risk factors for development and recurrence of primary breast abscesses. J Am Coll Surg 2010;211:41-8.

6. Agarwal MD, Venkataraman S, Slanetz PJ. Infections in the breast-Common imaging presentations and mimics. Semin Roentgenol 2017;52:101-7.

7. Huws AM, Semkin L, Moalla A, Udayasankar S, Holt SDH, Sharaiha YM. Primary squamous cell carcinoma of the breast in association with Zuska's disease. Breast Cancer 2018;25:365-9.

8. Kazama T, Tabeo I, Sekine C, Funamizu N, Onda S, Okamoto T, et al. Subareolar breast abscess in male patients: A report of two patients with a literature review. Surg Case Rep 2017;3:128.

9. Fu P, Kurihara Y, Kanemaki Y, Okamoto K, Nakajima Y, Fukuda M, et al. High-resolution MRI in detecting subareolar breast abscess. AJR Am J Roentgenol 2007;188:1568-72.

10. Crowe DJ, Helvie MA, Wilson TE. Breast infection. Mammographic and sonographic findings with clinical correlation. Invest Radiol 1995;30:582-7.

11. Guadagni M, Nazzari G. Zuska's disease. G Ital Dermatol Venereol 2008;143:157-60.

12. Hook GW, Ikeda DM. Treatment of breast abscesses with US-guided percutaneous needle drainage without indwelling catheter placement. Radiology 1999;213:579-82.

13. Karstrup S, Solvig J, Nolsoe CP, Nilsson P, Khattar S, Loren I, et al. Acute puerperal breast abscesses: US-guided drainage. Radiology 1993; 188:807-9.

14. Giess CS, Golshan M, Flaherty K, Birdwell RL. Clinical experience with aspiration of breast abscesses based on size and etiology at an academic medical center. J Clin Ultrasound 2014;42:513-21.

15. Li S, Grant C, Degnim A, Donohue J. Surgical management of recurrent subareolar breast abscesses: Mayo Clinic experience. Am J Surg 2006;192:528-9. 